\title{
Supine acceptance of a conventional imaging position may make you less prone to success
}

\author{
Guido Germano, PhD, ${ }^{\text {a }}$ Piotr J. Slomka, PhD, ${ }^{a}$ and Daniel S. Berman, MD $^{\text {b }}$
}

Cardiac SPECT imaging, like most medical imaging employing planar and tomographic data acquisition techniques, has traditionally been performed with the patient in the supine position, i.e. lying face up on the imaging bed.

It is easy to understand that this position, while convenient from a procedural standpoint, may not be the most comfortable for all patients. Several studies suggest that the side-lying position is by far the most common adult sleep position. ${ }^{1-3}$ The prolonged imaging times in the supine position, with arms held awkwardly over the head, frequently results in significant patient motion, reported between $26 \%$ and $32 \%$ of the time. ${ }^{4,5}$ Moreover, supine SPECT imaging is often associated with attenuation artifacts, commonly affecting the anterior myocardial wall in females and the inferior wall in males.

Prone SPECT imaging was proposed by Esquerré et $\mathrm{al}^{6}$ as a means to reduce inferior wall attenuation in 201-Tl studies, and was later found by Kiat et $\mathrm{al}^{7}$ to improve the overall specificity for the detection of coronary artery disease in a male population. In both of those studies, dedicated imaging tables with cutouts were used to reduce the effect of table attenuation, though nowadays that is no longer considered necessary due to the use of new table materials.

Of course, it is well understood that imaging a patient in the prone position only changes the relative position of the heart and the attenuating structures that surround it (breasts, diaphragm, etc.), and therefore prone imaging does not solve the problem of attenuation; rather, it changes the manner in which it affects the final images. In addition to changing the attenuation patterns in the anterior and inferior walls, a major appeal of the prone imaging approach is its demonstrated

\footnotetext{
From the Department of Medicine, ${ }^{\mathrm{a}}$ and Department of Imaging, UCLA School of Medicine, Cedars-Sinai Medical Center, Los Angeles, CA.

Reprint requests: Guido Germano, PhD, Department of Medicine, UCLA School of Medicine, Cedars-Sinai Medical Center, Los Angeles, CA; germano@aim.csmc.edu.

J Nucl Cardiol 2010;17:16-8.

$1071-3581 / \$ 34.00$

Copyright (C) 2009 by the The Author(s). This article is published with open access at Springerlink.com

doi:10.1007/s12350-009-9187-5
}

ability to substantially reduce the incidence of patient motion, thanks to the anterior chest wall (with the heart immediately beneath) being in direct contact with the table and to the more comfortable arm position, usually maintained by folding the arms under the patient's head. $^{7}$

Shin et al have recently suggested that the diagnostic performance of a prone-only SPECT imaging approach is quite comparable to standard supine SPECT imaging, with sensitivities of $88 \%$ and $92 \%$ for detecting coronary artery stenoses $\geq 50 \%$ and $\geq 70 \%$, respectively, and a normalcy rate of $95 \%$ obtained by visual expert analysis. ${ }^{8}$ Our group has demonstrated that prone images are associated with different normal count distributions compared to supine images, ${ }^{9}$ similar to the differences between attenuation-corrected and non-corrected studies; therefore, interpreting physicians must be aware of these differences and learn how to read prone SPECT images. Computer quantification of myocardial perfusion prone images can be helpful in this regard and can be automatically accomplished through the use of pronespecific normal limits..$^{10,11}$

Of note, it is important to realize that prone imaging is only a subsample of "non-supine" SPECT imaging, which has increased significantly with the advent of newer, small footprint cameras in which the patient is either sitting or reclining as if in a dentist chair. ${ }^{12}$ Efforts are ongoing to ascertain the relative performance of supine and non-supine SPECT in terms of quantitative results and diagnostic/prognostic assessment, and it is likely that some intrinsic differences will be found and will be difficult to eliminate.

Our professional societies' approach to prone imaging has been one of caution-as Shin et al point out, "recent MPS guidelines recommend prone imaging only in combination with supine imaging, and caution against performing prone imaging alone." Of course, combining supine and prone imaging increases the duration of the overall study, a matter of some concern at a time of declining reimbursement. At Cedars-Sinai, prone SPECT is routinely performed immediately after supine SPECT, but for a shorter time compared to the supine study (15 seconds/stop vs 25 seconds/stop). ${ }^{9}$ While we tried to perform prone imaging alone as a protocol for a year in the past, we found that there was still uncertainty regarding soft tissue attenuation and 
noted that there was commonly an anterior wall attenuation artifact on prone imaging not seen on supine imaging. When the combined supine/prone imaging became our routine, we found that our ability to identify attenuation artifacts increased, as we were able to require that a perfusion defect be present in both positions for the underlying myocardium to be considered abnormal. We also found that observer confidence in interpretation increases significantly when the two sets of images are available for analysis, compared to having a single set in either the supine or prone positions.

To aid in the implementation of combined supine and prone imaging, computer software has recently been developed that automatically does what the expert reader does in requiring defects to be present in both positions to be considered "real", ${ }^{11}$ To start, separate prone and supine gender-specific normal limits are derived from 40 males and females with low likelihood of CAD and are applied to compute hypoperfusion extent and severity on separate prone and supine polar maps. The approach considers hypoperfusion abnormalities to be significant only when detected on both supine and prone images at the same polar map location, with some exceptions for anterior and apical wall in males (where supine-only hypoperfusion was considered) and inferior wall (where prone-only hypoperfusion was considered). Finally, a combined Total Perfusion Deficit value is computed by the software, incorporating data from both scans. ${ }^{9}$ This approach proved able to eliminate most spurious defects, which occur only on one of the scans, and is conceptually applicable not only to photon attenuation artifacts but to several kinds of artifactual defects (e.g. motion-related), which may be present in prone or supine studies.

Comparison between supine, prone, and combined quantification for detecting angiographically significant CAD in 369 consecutive patients demonstrated that the combined approach yielded significant gains in specificity ( $86 \%$ vs $65 \%$ ) and accuracy ( $86 \%$ vs $79 \%$ ) over supine-only quantification, without compromising sensitivity ( $85 \%$ for both supine and combined methods). ${ }^{9}$ In a subsequent study, this approach was separately validated in a female population, where breast tissue causes specific photon attenuation effects. ${ }^{11}$ A population of 168 women with correlating angiography and 291 with a low likelihood of CAD was studied, and it was found that the combined prone-supine quantification approach increased specificity for CAD detection to $94 \%$ from $61 \%$ (supine) and $76 \%$ (prone), without decrease in sensitivity. Normalcy rates were also significantly higher $(97 \%)$ compared to supine-only $(85 \%)$ and prone-only (91\%) imaging, with the largest improvements corresponding to the largest breast sizes. We found that most of the reduction in false positive rate obtained by the combined prone-supine approach is due to "shifting breast" attenuation discrepancies between the supine and prone positions, which were correctly recognized as artifacts by the combined quantification method. In this context, it should be noted that there is no study to date demonstrating that myocardial perfusion SPECT with attenuation correction improves accuracy for detection of CAD in women, despite a wealth of literature on attenuation correction in general. In further validation, the combined prone-supine approach was also applied to an obese population ${ }^{13}$ where it demonstrated incremental diagnostic value for predicting $50 \%$ or greater and $70 \%$ or greater coronary artery stenosis.

It is interesting to note that a recommendation similar to that in favor of combined supine/prone SPECT imaging has been made by the societies with respect to attenuation-corrected SPECT, which is supposed to only be used for myocardial perfusion assessment together with non-attenuation corrected SPECT images. ${ }^{14}$ While attenuation correction, with either supine or prone imaging, would be a more direct way of diminishing the effect of variable soft-tissue attenuation, it lengthens study time compared to single view imaging due to imaging, set-up, and quality control times, adds cost for the hardware and does not result in higher reimbursement. ${ }^{15}$ By the end of 2002, fewer than $10 \%$ of SPECT cameras sold worldwide were equipped with AC systems, and as recently as 2005 it was reported that "the entire base of SPECT cameras has only $5 \%$ of systems with attenuation correction.", 16

What to do, then? Is a back-to-back supine/prone acquisition strategy intrinsically undesirable, or can we accept prone imaging alone as providing more accurate patient assessment than supine imaging? Our opinion is that it may help to think of the matter as of a natural evolution of nuclear cardiac imaging. In the old days, multiple planar views were preferred to a single viewthis of course evolved into SPECT, which in turn evolved by adding a fourth dimension (time, as in gated SPECT). One could conceivably say that we are once again upgrading our technique, this time to "multiple SPECT views". The extra time required for the supine + prone approach may well be reduced by newer faster cameras and reconstruction algorithms, ${ }^{12}$ just like SPECT and gated SPECT were made practical by faster computers and radiopharmaceuticals with more favorable uptake characteristics.

Combined supine/non-supine or multi-view SPECT is of course also conceptually applicable to many of the new "fast" cameras using non-conventional imaging stances. High-speed multi-view (semi-upright and supine) perfusion SPECT imaging has been recently described, ${ }^{17}$ and quantitative analysis similar to our 
combined prone supine approach could be therefore developed for these imaging protocols as well, since it is conceivable that similar effect of shifting attenuation artifacts will be present in this imaging configuration.

Ultimately, we understand that the economics of healthcare and consideration of patient throughput and reimbursement patterns are bound to steer (hopefully not stunt) the evolution of SPECT... but it is our opinion that prone imaging, particularly when it is combined with supine imaging, represents a very useful tool that can increase our chance of success, where "success" is defined as obtaining the most accurate possible assessment of the patient with the greatest interpreter confidence, given one's equipment and clinical acumen.

\section{Open Access}

This article is distributed under the terms of the Creative Commons Attribution Noncommercial License which permits any noncommercial use, distribution, and reproduction in any medium, provided the original author(s) and source are credited.

\section{References}

1. Johnson HM, Swan TH, Weigand GE. In what positions do healthy people sleep? J Am Med Assoc 1930;94:2058-62.

2. Dekoninck J, Gagnon P, Lallier S. Sleep positions in the youngadult and their relationship with the subjective quality of sleep. Sleep 1983;6:52-9.

3. Gordon S, Grimmer K, Trott P. Self reported versus recorded sleep positions: An observational study. Internet J Allied Health Sci Pract 2004;2. http://ijahsp.nova.edu/articles/Vol2num1/Gordon_ sleep.htm.

4. Botvinick EH, Zhu YY, O'Connell WJ, Dae MW. A quantitative assessment of patient motion and its effect on myocardial perfusion SPECT images. J Nucl Med 1993;34:303-10.

5. Prigent FM, Hyun M, Berman DS, Rozanski A. Effect of motion on thallium-201 SPECT studies: A simulation and clinical study. J Nucl Med 1993;34:1845-50.
6. Esquerré JP, Coca FJ, Martinez SJ, Guiraud RF. Prone decubitus: A solution to inferior wall attenuation in thallium-201 myocardial tomography. J Nucl Med 1989;30:398-401.

7. Kiat H, Van Train KF, Friedman JD, Germano G, Silagan G, Wang FP, et al. Quantitative stress-redistribution thallium-201 SPECT using prone imaging: Methodologic development and validation. J Nucl Med 1992;33:1509-15.

8. Shin JH, Pokharna HK, Williams KA, Mehta R, Ward RP. SPECT myocardial perfusion imaging with prone-only acquisitions: Correlation with coronary angiography. J Nucl Cardiol 2009;16: 590-6.

9. Nishina H, Slomka PJ, Abidov A, Yoda S, Akincioglu C, Kang $\mathrm{XP}$, et al. Combined supine and prone quantitative myocardial perfusion SPECT method development and clinical validation in patients with no known coronary artery disease. J Nucl Med 2006;47:51-8.

10. Patton JA, Slomka PJ, Germano G, Berman DS. Recent technologic advances in nuclear cardiology. J Nucl Cardiol 2007;14: 501-13.

11. Slomka PJ, Nishina H, Abidov A, Hayes SW, Friedman JD, Berman DS, et al. Combined quantitative supine-prone myocardial perfusion SPECT improves detection of coronary artery disease and normalcy rates in women. J Nucl Cardiol 2007;14:44-52.

12. Slomka P, Patton J, Berman D, Germano G. Advances in technical aspects of myocardial perfusion SPECT imaging. J Nucl Cardiol 2009;16:255-76.

13. Berman DS, Kang XP, Nishina H, Slomka PJ, Shaw LJ, Hayes $\mathrm{SW}$, et al. Diagnostic accuracy of gated Tc-99m sestamibi stress myocardial perfusion SPECT with combined supine and prone acquisitions to detect coronary artery disease in obese and nonobese patients. J Nucl Cardiol 2006;13:191-201.

14. Hansen C, Goldstein R, Akinboboye O, Berman D, Botvinick E, Churchwell K, et al. Myocardial perfusion and function: Single photon emission computed tomography. J Nucl Cardiol 2007;14:e39-60.

15. Germano G, Slomka PJ, Berman DS. Attenuation correction in cardiac SPECT: The boy who cried wolf? J Nucl Cardiol 2007;14:25-35.

16. Hendel RC. Attenuation correction: Eternal dilemma or real improvement? Q J Nucl Med Mol Imaging 2005;49:30-42.

17. Berman D, Kang X, Tamarappoo B, Wolak A, Hayes S, Nakazato R, et al. Stress thallium-201/rest technetium-99m sequential dual isotope high-speed myocardial perfusion imaging. J Am Coll Cardiol Imaging 2009;2:273-82. 